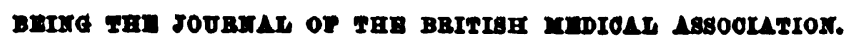

EDTTED BY ANDREW WYNTER, M.D.

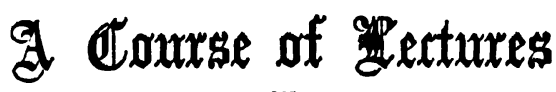 \\ ors}

\section{THE NATURE AND TREATMENT OF DEFORMITIES.}

DELIVERED AT THE ROYAL ORTHOPADIO HOSPITAL, MAX 1860.

$$
\text { Br }
$$

RICHARD WM. TAMPLIN, F.R.C.S., SUhoroR TO THE hOSPITAL.

Lecture II (concluded from page 516).

TALIPEg EqUINU8.

Tae next condition is one, the real cause of which is totally unknown to us. Suddenly, without any apparent illness before or afterwards, a child is deprived of the use of one or both extremities, sometimes of the whole of them. Numerous cases have presented themselves, the history of which is as follows : The child went to bed in perfect health, passed a good night, but on being taken up in the morning, was found to have lost the use of one or both lower extremities; upon partial recovery from which, contraction of the gastrocnemius remained. That there is some organic affection of the spinal marrow or its membranes in these cases, is more than probable; and I be. lieve that, if opportunities were presented of careful examin. ation with the assistance of the microscope, it might be disco. vered.

Upon one occasion, I was consulted by a lady, 22 years of age, who had been attacked eleven years previously with contraction of both elbow.joints at right angles, and of both the gastrocnemii muscles. Her mother stated, not only that she enjoyed good health at the time, but that she had always possessed good health both before and since the attack. In this instance, there was not complete loss of power in any of the muscles, so that the mischief existed in a less degree. In other instances-in healthy labourers, for example-a gradual lameness comes on from contraction of the gastrocnemius muscle, without paralysis, or any apparent interference with the voluntary efforts, the health remaining uninterruptedly good. We are therefore evidently in great ignorance upon this subject; and when we consider that the contraction itself was but a few years since, comparatively speaking, regarded as incurable by the leading members of our profession, we have reason to hope that, having arrived at a successful mode of treatment, the cause may yet be discovered.

There is another cause with which I have met; viz., Fracture of the Tibia low down. One patient was aged 40 years, and another was 60 years of age. In both these, the cons, had evidently taken place during the treatment of the fracture The first case, which had existed four years, had become permanent from want of exercise of the joint; and in the other, the same result would in all probability have followed if sufficient time had elapsed. I divided the tendo Achillis in the former case, and restored motion. The latter having only existed ten or twelve months, by the use of the Scarpa's shoe the contraction was overcome, and voluntary motion restored.

In all cases, from whatever cause they arise, a greater or less amount of atrophy of the affected limb will be found to exist, owing to the necessarily passive state of the joint. In the paralytic cases, there is a total absence of development; those muscles which are paralysed degenerating into a sort of fatty substance, whilst those which are contracted suffer from the absence of exercise.

In those cases in which paralysis exists, of course any after development or increase in the size of the limb is out of the question; but in those in which the muscles retain their func. tions after the removal of the contraction and restoration of the motion of the joint, the muscles, by proper exercise, will increase in development. Two patients, 38 years old, who had suffered from this deformity frum their childhood, and upon whom I operated more than fourteen years since, now possess increased development and perfect power over all the muscles, having had no assistance or support to the joint after the first fow months following the treatmont.

There are other cases which, strictly speaking, are simply talipes equinus, where the deformity is considerably aggravated in appearance, without much additional resistance or obstacle to the treatment. (Figs. 3 and 4.) These cases result from

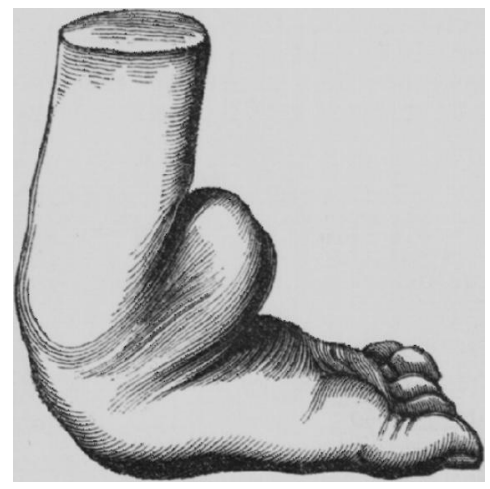

Fig. 3.

Paralysis of the whole of the Muscles situated in the front part of the Leg, connected at the onset with paralysis of the entire extremity. When, upon partial recovery, the patient begins to use the limb, the toes of the foot, which is completely extended (as seen in Fig. 1), are slightly flexed from the loss of power in the extensors; and these being subjected to pressure on their superior anterior surface by the weight of the body in walking, the foot, from having no support excepting that derived from the ligaments and the tendo Aohillis, deviates to one side or the other, generally inwards. By degrees, the ligaments, as in every case where they are subjected to undue pressure, become stretched, giving rise to further deviation, which adds considerably to the deformed appearance of the foot (see Fig. 4); the patient walking at last upon the outer

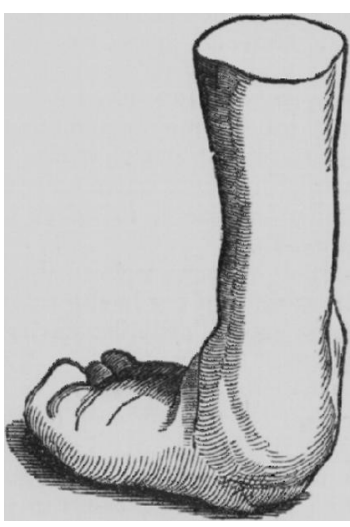

Fig.4.

and upper surface of the foot, as in complete talipes varus; and there are instances of complete reversal of the anterior half of the foot-the toes pointing backwards, the sole up. wards, and the patient walking upon the dorsum of the foot, as 
seen in Fig. 3. The muscles do not of necessity - at least, but seldom-present any obstacle to the restoration of the natural position of the foot, beyond the contracted gastrocnemius.

The ligaments in thesc, as in all paralytic cases, partake of the general debility, and offer but little resistance to a change of position. We have had patients from thirty to forty years of age, who had suffered since infancy from this deformity in the above severe forms, yet have had their feet restored to the normal position with the power of walking in comfort with the assistance of mechanical supports attached to their boots.

Contraction of the Gastrocnemius in the slightest form-viz., that in which the foot is at a right angle, the patieut being able to stand flat upon it-must be regarded as an incomplete form of talipes equinus. Even this is a serious inconvenience, as the pressure of the weight of the body is thrown upon the anterior inferior extrenity of the metatarsal bone, giving rise to corns and other annoyances, such as a sudden deviation of the foot to either side when any inequality of su:face is trodden upon. The weight is then thrown on one or other of the lateral ligaments, which occasions severe pain, preventing at times the use of the foot for twenty-four hours. The ligaments become weakencd by this sinccession of sudden strains, and remain in a stretched condition, which of course produces greater liability to a recurrence of the casualty.

Contraction of the great toe is commonly a sign of contraction of the gastronnemius at right angles, especially in the adult, as shewn in Fig. 5. It arises from the extensor muscles being instinctively brought into action to assist the flexors of the foot to bend the joint-a secondary effect, which, it will be seen, cannot be produced until the toe is extended.

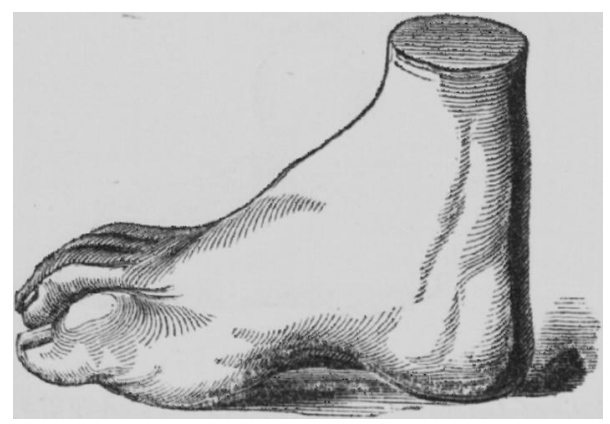

Fig. 5.

Symptoms. The natural temperature of the limb is reduced in all cases, but more especially in those connected with paralysis. The passive condition of any joint or extremity predisposes the limb to a reduction of the natural temperature. Many suffer se. verely from chilblains during the winter. One case upon which I operated some years since, had for two years an open wound extending completely across the front part of the font, pro. duced through this inability to preserve the natural tempera. ture, which cicatrised perfectly in three weeks, without any other application than simply wrapping the foot in sheets of wadding. I have a jatient non in the Hospital with paralytic talipes equinus, of a severe form (precisely resembling Fig. 4), affecting both feet, who had open wounds upon both ankles for upwards of four months. Stimulants and ointments, the girl states, had been constantly applied by medical advice, with. out any benefit, the ulcer remaining in its sluggish, unhealthy state, without any attempt at cicatrisation. The simple appli. cation of sheets of wadding and warm covering sufficed in this, as it will in all similar instances, to effect perfect cicatrisation in less than three weeks.

It has been asserted by some writers upon this subject, that an alteration at times takes place in the relative size or propor. tion of the astragalus and its articular cavity. I do not believe that this ever happens. The resistance offered in old cases to the return of the astragalus within its articular cavity, is owing more to the absence of the secretion of the synovial membranc and to the rigidity of the ligament. In not one instance have I seen a failure in the attompt to remove the deformity, although I have had to treat these cases in patients upwards of fifty years old. This could not be if the astragalus were too large or the articular cavity too small.

Treatment. 'The trentment is the next consideration; and although this is, as I have stated to you, one of the most simple forms of contraction, yet there are points which will demand your careful attentions.
In all cases in which there is decided contraction of the gastrocnemins, although it will admit of flexion to a right angle my advice is that the tendon should be divided. Attention must, however, be directed to the oause of the deformity, its duration, and the age and constitution of the patient.

In cases which have arisen from the three first named causes, and in which there has been, or may be at the time, a loss of power, with complete paralysis of one or more muscles, there will be found no resistance in the joint itself, the deformity being maintained solely by the contraction of the muscle. You will therefore at once perceive that, if the patient is young and of delicate constitution, the division of the tendo Achillis will admit of the foot being placed in any position without difticulty; and if the foot is at once or within a few days tlexed, there is great danger of producing an irremediable deformityviz., talipes calcaneus or talipes calcaneo-valgus. I have boen applied to in cases in which this deformity had been thus produced. The uniting medium, having been too rapidly stretched, had lost its power of recontraction, and a permanently elongated tendon was the result; so that, although the muscle retained its functions, yet, before it could act on the os calcis, it contracted fully upon itself. The heel thus loses its support, and one or other of the above mentioned deformities is produced.

I must caution you against a practice which is now being recommended; viz., that of dividing tho tendons, and imme. diately placing the foot or joint in position. It is a violation of all principles of treatment, and must be followed at times by irremediable consenuences.

To preserve the uniting medium in slight cases, and to render the tendon available hereafter, a slow extension must be aclopted; and in the paralytic cases there is always considerable weakness existing for three or four months after the operation. In consequence, as I imagine, of this, it has been the practice of some to avoid the operation in slight cases. I find, however, with the precaution I adopt, not only that the operation is not injurions, but that it is the only mode by which relief can be obtained.

The manner of performing the operation is as follows :-Let the patient lie on the abdomen; an assistant grasps the knee of the leg to be operated upon with one hand, and the foot with the other, the leg being kept extencled; direct him forcibly and steadily to flex the foot, so that the tendo Achillis, upon which jou nre about to operate, is licpt at the full stretch; then introduce, about an inch and a half or two inches above the 08 calcis, a small sharp.pointed knife, pass it horizontally beneath the tendon, turn the sharp edge of the knife towards the ten. don, and, by gently pressing the tendon upon the edge of the lnife with the thumb or finger of your left hand, gradually divide it from below upwards, and the moment the teudon is divided, let the assistant hold the foot in the full extended position, that the divided ends may be kept as closely as possible in juxtaposition. A piece of lint and strapping must then be applied over the puncture, the foot carefully bandaged from the toes; two splints, either of gutta percha or tin softly padded, being applied one in the front, and the other on one side of the foot, retained in their position by the bandage, and holding the foot in the full extended position. The leg should be kept at rest in the horizontal posture, and be carefully wrapped up in wadding and flannel to preserve the natural temperature; the ordinary diet should not be interfered with; and the only caution to bo given to the patient is, that during any movement the leg slould be held in the horizontal position, for the first few days. In the cuurse of from four to seven days, you commence your exten. sion, provided the puncture is healed. The best instrument is the Scarpa's shoe, with the single or the double action cogwheel (Fig. 6), which I have used for many years. After re. moving the splints and strapping, let the foot be rebandaged with flannel, the Scarpa's shoe be screwed to an angle precisely corresponding with the position of the foot, which is then to be placed in the instrument and secured by the ankle and instep straps, avoiding pain and undue pressure. You then proceed with your extension, in slight cases, slowly; occupying at least fourteen days in bringing the foot to a right angle, and as many more in gaining the full Hexed position. The patient muy ther be allowed to move about and use the foot moderately in the instiument, which must not be removed for six weeks from the time of the operation, when an ordinary boot may be used in those cases in which there is no paralysis or lateral deviation: but in cases where these conditions exist, a boot with iron support attached to each side (Fig. 7 ), with stop.joint, to prevent extension beyond a right angle in paralytic cases, must be worn for a longer or shorter period, azcording to the strength 
gained. I use the double iron, in order that the foot mey be enpported on cither side, at the tesdency to deriation may and does alternate in paralytic cases. In some cases, where there had been perfect puralysis of the anterior tibial muscle, the patient has, in the course of a few years, gained suficien strength in this ligaments to dispense with support for all ordinary exerciser.

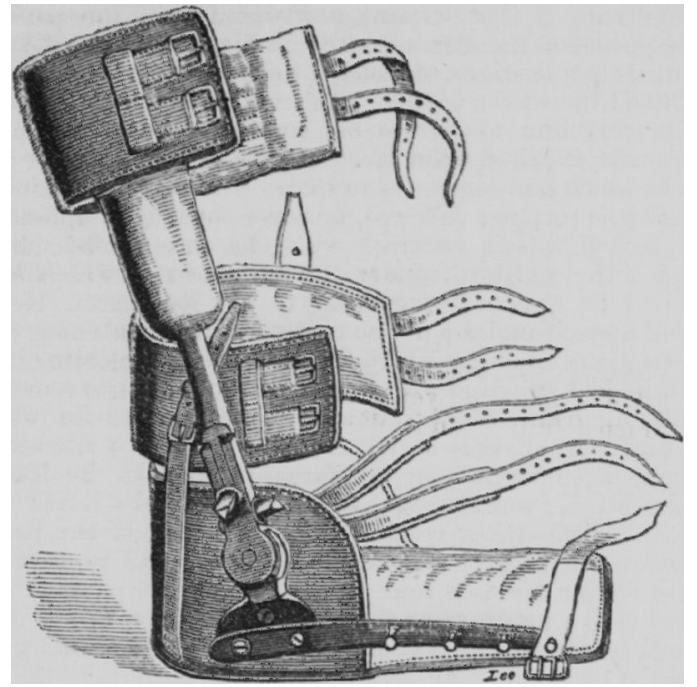

Fi.. 0.

Many cases of this kind have been successfully treated, the patients retaining the full power in the gastrocnemins, although they had shewn the greatest possible weakness immediately after the treatment, the joints appearing almost unattached.

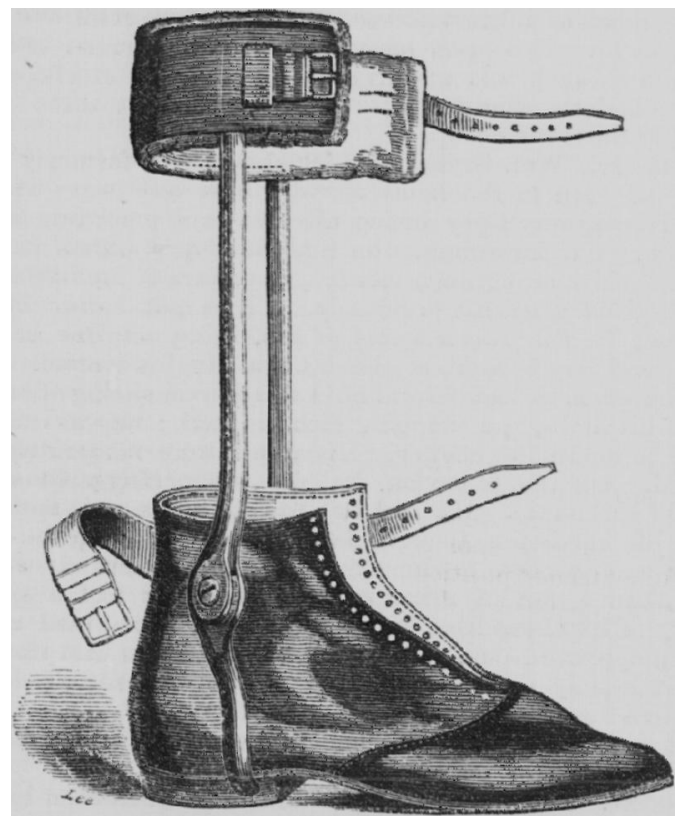

Fig. 7.

Care must be taken to preserve the natural temperature of the limb in all these cases, or the healing of the puncture and union of the tendon will certainly be retarded, and even prevented. With only ordinary covering, the tempernture will be below the natural and healthy standard, and the restorative process cannot go on. A medical gentleman divided the tendo Achillis for talipes equinus, and, with the view of preventing inflammation, applied cold lotions over and around the puneture. After fourteen days, he found the puncture still open, and no attempt at union. He wrote and informed me of the particulars. I advised him to wrap the foot and ler in flannel, etc., and keep the limb at rest. In four or five days, perfect union took place, and he proceeded with his extension, which nust in no case be commenced until the juncture is healed.
My reason for advising the operation in these slight cases is, that I hare never seen the use of the joint restored without it. Patients have applied to me aftor having been treated mechanicully for years, and by some who are considered authorities upon this subject, with strict injunctions never to allow an operation to be peiformed, as its only result would be increased lameness. I have operated in these cases, and have had the satisfaction of seeing the patient walk, with the free use of the joint, in six or seven weeks, with the assistance, of course, of support to the ankle, which they were required to wear for some months afterwurds.

In cascs which have arisen from rheumatism, there is no fear of your proceeding too rapidly. The difticuliy with which you will have to contend is the very reverse, namely, an inpossibility to proceed with the flexion of the joint as quickly as could be desired, during the time when the uniting medium is soft and clastic. There is always, in these cases, more or less thickening of the synovial membrane and parts surrounding the joint, which renders it very difficult to extend the astraga lus to its proper position in the articular carit $\because$, and this dif. ficulty is greatly increased by the pain occusioned at each alteration of the position of the astragalus, however slight. It, therefore, requires great resolution on the part of the patient, as well as perseverance on the part of the medical attendant. By steadily continued extension, however, the treatment may be borne witliont interruption to the general health.

In adult cases, which have existed many years, pain always attends a change of the position of the foot. This will not, however, offer an obstacle to the treatment, exceptiug in the rbeamatic cases just alluded to. You must at all times be guided ly the feelings of the patient, and it is better to progress slowly and certainly, than to attempt to proceed rapidly, and find yourself obliged to intermit the treatnent. In some cases of confirmed talipes equinus, which present no lateral devintion before the operation, you will find that, as you tlex the foot, lateral deviation will take place, most commonly to the outer side -rarely to the inner. This is owing to the contraction of the peronei muscles on the one hand, or the posterior tilial on the other, and is frequently not perceived until you have bronglit the foot to nearly a right angle with the leg. It is hetter then to intermit the treatnent, to divide the peronei or posterior tibial, as the case may be, and in three or four days resume the treatment: otherwise the patient, when beginning to walk, will be thrown either on the inner sicle of the metatarsal bone of the great-toe, or on the outer edge of the fifth metatarsal bone, occasioning him great pain and inconvenience. In no case must you rest satisfied with the simple removal of the deformity, but the foot must be flexed to nearly its fullest extent, or a second operation will be vecessary; and in severe cases the patient must wear the instrument night and day, in order to prevent the tendency to recontraction; for, from my observation, the cicatrix does not resolve itself into the permaneut condi tion in less than six months.

The increase of the arch of the foot, and the contraction of the toes, the grent toc cspecinlly (Fig. 5), will most cummonly vield to the stcaly and continued pressure which ought to be maintained by the straps passing over the instep. In old cases, however, it is sometimes necessary to divide the ficxor tendons of the toes.

Contraction of the arch of the foot is seldom met with, exrept in connection with contraction of the tendo Achillis, paralysis of the gastrocnemius, or congenital cases of contraction. It is well for you to bear this in mind, in slight cases, for I have lately scen the plantar fascia divicled simply for this de. formity-the cause, namely, slight contraction of the gastrocncmius, being entirely overlooled. Nivision of the plantar fascia is unnecessary, except in very few instances, and those only old cases.

Salate-Pox and Vaccination Hospital. A general court of the governors of this institution was held a few days ago. Sinco the foundation of the hospital, in 1746 , it has afforled relief to $273,(190$ patients; during the year 1850 , to $1,1 \times 5$; and during the past six months, to 660 -being the grcatcst uumber ad. mitted for a similar time since its institution. A tender has been accepted for building two additional wards to the hospital for the sum of $£ 3,358$, and these warils are to be procecded with at once. $1 \pm 7,73 \div$ persons have been vaccinated at the hospital since the year 1500 , and 197 during the last six months; and 120 medical practition ers have been supplied gratuitously with 600 charges of vaccine lym rh since the commencenent of the present year. 